\title{
Obstacles to Personal Creativity Among Arab Women from the Gulf Cooperation Council Countries
}

\author{
Ahmed M. Abdulla \\ Arabian Gulf University, Manama, Kingdom of Bahrain \\ E-mail address: ahmedmda@agu.edu.bh
}

Mark A. Runco

American Institute of Behavioral Research \& Technology, USA E-mail address: runco@uga.edu

\author{
Hanadi N. Alsuwaidi \\ Ministry of Education, UAE \\ E-mail address: h.n.alsuwaidi@ hotmail.com
}

Huda S. Alhindal

Arabian Gulf University, Manama, Kingdom of Bahrain

E-mail address: hudasah@agu.edu.bh

\section{ARTICLE INFO}

\section{Keywords:}

Creativity

Obstacles

Women

Arab culture

Gulf Cooperation Council countries

\section{Article history:}

Received 15 February 2018

Received in revised form 25 June 2018

Accepted 27 June 2018

ISSN: 2354-0036

DOI: 10.1515/ctra-2018-0003

\section{A B S TRACT}

Personal obstacles to creativity were investigated by sampling 297 Arab women from four Gulf Cooperation Council (GCC) countries: Bahrain, Kuwait, Saudi Arabia and the United Arab Emirates. The Obstacles to Personal Creativity Inventory, as self-report, was used. It assesses four types of obstacles (a) inhibition/shyness, (b) lack of time/opportunity, (c) social repression, and (d) lack of motivation. The results showed that the highest mean was reported for the lack of time/opportunities factor, followed in order by the three other factors: lack of motivation, inhibition/shyness, and social repression. (A high mean is indicative of more obstacles.) A multivariate analysis of variance indicated that reported obstacles to creativity significantly differed by field of study. Women in the arts reported experiencing fewer obstacles related to social repression in comparison with women in engineering, who showed the highest mean. No significant effects were observed for level of education, country and income in the GCC countries. The MANOVA also showed significant interactions between (a) education and sector (i.e., government vs private), (b) country and sector, (c) income and field of study, and finally (d) between field of study and sector. Results from this study were compared to two other studies, in Brazil and Mexico, that used the Obstacles to Personal Creativity Inventory. The high mean found for the lack of motivation in GCC countries deserves further investigation, given that motivation is so important for creativity and often is something that can be encouraged.

\section{INTRODUCTION}

There are surprisingly few theories about women's creativity or how to understand creative women. As Reis (2002) reported "little is known and limited research has been com- 
pleted about creative women, their creative processes, and the decisions they face about their own creative productivity" (p. 305). After nearly two decades of this predicament, nothing noteworthy seems to have changed. Simonton (2000) observed,

Psychologists still have a long way to go before they come anywhere close to understanding creativity in women and minorities. So far, creativity in such groups seems to display a complex pattern of divergence and convergence relative to what has been observed in majority-culture male study participants. (p. 156)

The limited knowledge on women's creativity is not limited to Western research. Very few investigations have aimed to understand women's creativity in other cultures. In fact, searching for literature on creative women in the Arab culture, or more specifically, the Gulf Cooperation Council countries ${ }^{1}$ (GCC), showed that women and creativity were not studied at all. The present research focuses on the creativity of GCC women. We begin by describing their cultural background and social context and by identifying challenges they might encounter. This is then related to gender differences in creativity, the focus being on the important distinction between creative potential and creative achievement.

\section{WOMEN IN THE GCC COUNTRIES}

Women in GCC countries share some commonalities with women in other cultures, such as experiencing a lack of time and opportunities, often valuing relationships over creative work, and prioritizing endeavours that put the family first (Reis, 2002). However, women in the Gulf region have their own unique challenges related to their social context. Difficulties in travelling without permission from their families, limited opportunities for training and skill development, lack of social support, and not being able to make major decisions in their life--such as whom they are going to marry--are just a few examples of challenges faced by GCC women (Al-Ahmadi, 2011; Taqi, 2016). Noticeably, efforts have been made by the GCC governments in the last two decades to offer social support for women. This support includes creating or modifying legislation to increase women's engagement in society. For example, Saudi women were recently (June 2018) allowed to drive for the first time in history. Another facet of governmental support is evident in the establishment of official institutes to ensure equal opportunities for women (e.g., the Supreme Council for Women in Bahrain and the Dubai Foundation for Women and Children in the UAE). Moreover, GCC governments have made great effort to ensure equal opportunities for women in education and scholarships; therefore, it is not surprising that women comprise

\footnotetext{
${ }^{1}$ The Gulf Cooperation Council (GCC), is a political and economic alliance of six countries: Saudi Arabia, Bahrain, Kuwait, United Arab Emirates, Oman and Qatar.
} 
about $60 \%$ of the graduates from Gulf universities (Coffman, 2003). However, despite this support for women's education, women in the GCC represent only $25 \%$ of the labour force, according to the World Bank ${ }^{2}$. As in some Western countries, women's underrepresentation in the work force is largely due to parent's expectations for their daughters; that is, women should get married and have children (Reis, 2002). This is in turn related to the fact that women in the GCC might have less time to engage in creative work. According to a United Nation's (2017) report, the average household size in the GCC ranged between 5.3 (in Qatar) to 8 (in Oman), compared with 2.1 in Germany, 2.3 in the United Kingdom and France, and 2.6 in the United States. Thus, time for creative engagement seems to be a major challenge facing GCC women.

To summarize, women in the GCC have more support now than ever; they are highly educated, although society might not fully and directly benefit from its investment in women's education due to some cultural expectations. Lack of time seems to be an issue due to the norm for large household size, which characterize GCC families. As in Western culture, women in the GCC are expected to take care of extended family members. Thus, an aspect of the current study was to compare married vs. single women. The hypothesis is straightforward: single women would report fewer obstacles related to time than married women.

As noted above, women in the GCC share some common challenges and barriers with women in other countries. GCC women also might face unique challenges related to their social context. This study aimed to explore four kinds of personal obstacles among GCC women, which will be specified after a brief discussion of the literature on gender differences in creativity.

\section{GENDER DIFFERENCES IN CREATIVITY: POTENTIAL VS. ACHIEVEMENT}

Previous research on gender differences in creativity underscores the importance of the distinction between creative potential and creative achievement. Some previous research has investigated gender differences in creative potential, as assessed by various paper and pencil tests (e.g., divergent thinking tests). They uncovered very few significant differences between men and women in creative potential. That is, only one-third of those studies reported significant differences favouring females (Runco, Cramond, \& Pagnani, 2010). In contrast, studies that considered achievement as a criterion of creativity showed large gender differences favouring males (Baer \& Kaufman, 2008). There is no single answer or explanation why males outperform females in creative performance, although studies of creative achievement typically cover decades (e.g., Cattell, 1903; Diamond,

\footnotetext{
2 https://www.worldbank.org/en/news/feature/2017/09/29/why-supporting-womens-economic-inclusion-is-
} vital-for-the-gcc 
1986; Simonton, 1984, 2016, 2018). Certainly, throughout history, females have had educational and resource disadvantages. Cramond (2011) gave examples of women from different parts of the world who have had unequal access to education and specialized training compared to males. She concluded: "One of the many barriers for women has been the inaccessibility of the education they need to progress in many fields, including the sciences" (p. 522). Another explanation found in the creativity literature is that males share higher variance in their creativity compared to females (He \& Wong, 2011; Karwowski et al., 2016). According to the greater male variability in creativity hypothesis, "Among men, there likely will be more poorly, but also more highly creative individuals than among women" (Karwowski et al., 2016, p. 467).

Creative performance can be defined as "The actual transformation of creative abilities into a measurable achievement or product" (Pagnani, 2011, p. 551). This definition leads to an operational question, namely, what factors might hinder women from transforming their creative abilities into a measurable product or recognized achievement?

Previous works on barriers to creativity have investigated a wide range of obstacles, including those related to organizations (e.g., Mostafa \& El-Masry, 2008; Rickards \& Jones, 1991; Rosenberry, 2005; Sadi \& Al-Dubaisi, 2008), work environment (e.g., Isaksen, Lauer, Ekvall \& Britz, 2000-2001; Walter, 2012; Witt \& Boerkrem, 1989), and personal situations (e.g., Alencar, 2001; Alencar, Fleith \& Martinez, 2003; Reis, 2002). The current study focused on the personal obstacles to creativity in a population that has been rarely studied: women from the GCC countries.

Personal obstacles to creativity might be classified in various ways, some of which are external, including(a) lack of opportunities, (b) the culture in which women mature, and (c) the social expectations of the society in which they live (Reis, 2002). Examples of internal obstacles include (a) lack of motivation, (b) inhibition, (c) prioritizing (i.e., career vs. personal life), and (e) some personality characteristics such as selflessness, shyness, modesty, lack of self-confidence and self-esteem, loneliness, and isolation (Alencar, 2014; Reis, 2002).

The present investigation differs from most of the previous work on creativity, even those conducted in Western culture, by the sampling of women from several different countries and the inclusion of educational levels, socioeconomic status, and field of study in the analyses. This is in addition to what was mentioned earlier: the majority of the previous studies on gender differences in creativity has focused on the large gap between males and females in terms of creative productivity. The majority of those studies were conducted on participants from Western cultures, with few studies conducted outside 
of Western, educated, industrialized, rich, and democratic cultures (WEIRD; Karwowski et al., 2016). There is a paucity of studies that sampled Arab culture, especially the Gulf region. Thus, the current study aimed to answer the following questions:

What are the most notable obstacles to personal creativity as perceived by adult females in GCC countries?

Are there any differences among the different kinds of obstacles, and are these related to country, age, level of education, field of study, marital status, income, and (government vs private) sector?

\section{METHOD}

\section{Participants}

The study sample consisted of 297 women representing four countries: Bahrain (17.5\%), United Arab Emirates (31.3\%), Saudi Arabia (35.7\%), and Kuwait (15.5\%). The participants' ages ranged from 18 to 61 years old, with a mean age of 35.9 ( $S D=8.34$; $M d n=36)$. Regarding education, the majority reported studying or holding an undergraduate degree 171 (57.6\%), with 113 (38\%) reported studying or holding a master's or a doctoral degree, and 13 (4.4\%) reported holding a high school degree. Monthly income was as follows: $42(14.1 \%)$ reported earning a monthly salary of 600 Bahraini Dinar $(\approx \$ 1,600)$ or below, with $79(26.6 \%)$ earning between 601 and 1,200 BD $(\approx \$ 1,600$ $\$ 3,200), 71$ (23.9\%) earning between 1,201 and 1,800 BD ( $\approx \$ 3,200-\$ 4,700)$, and 105 (35.4\%) earning more than 1,800 BD monthly ( $\approx \$ 4,700$ and above). Most of the participants indicated that they were married $(n=198,66.7 \%)$ and $99(33.3 \%)$ indicated they were single. The participants were from six different fields of study: 145 (48.8\%) social sciences, 56 (18.8\%) hard science, 46 (15.5\%) business, 29 (9.8\%) engineering, $13(4.4 \%)$ mathematics, and $8(2.7 \%)$ the arts. The majority $(78.8 \%)$ indicated that they worked in the public sector, while $53(17.8 \%)$ indicated that they worked in the private sector. Ten (3.4\%) indicated that they had their own business.

\section{Instruments}

The Obstacles to Personal Creativity Inventory (Alencar, 2014) was used to identify four types of blocks to creativity among the GCC women. The inventory contained 66 items. A 5-point Likert scale was used to allow the participants to express how much they agree or disagree with a particular statement: from 1 (totally disagree) to 5 (totally agree). The questions assessed four factors: (a) Inhibition/Shyness, (b) Lack of Time/Opportunities, (c) Social Repression, and (d) Lack of motivation.

The Inhibition/Shyness factor included 23 items related to the "emotional nature that block the expression of personal creativity" (Alencar, 2014, p. 27). The Lack of Time/ 
Opportunity factor consists of 14 items related to the "limited availability of time, resources, and opportunities to express the potential to create" (p. 27). The third factor, Social Repression, consists of 14 items "concerning different factors of a social nature that block creativity" (p. 29). The fourth factor, Lack of Motivation, "includes 20 items that relate mainly to the absence of personal motivation elements that facilitate the expression of creativity" (p. 30). Each of the 66 items starts with: I will be more creative if....Alencar (2014) reported that all internal-consistency coefficients for the instrument were greater than .85. The second instrument used for this study was a demographic questionnaire. It asked about participants' age, education, field of study, nationality, income, and sector (government, private).

\section{Procedure}

The Obstacles to Personal Creativity Inventory was translated from English into Arabic by the first author who is a native speaker of Arabic. The translated version was then checked by two bilingual experts and minor corrections were made. The data were collected in collaboration with official authorities in the four countries, including the Supreme Council for Women, the Supreme Council of Family Affairs, and the National Council for Culture, Arts, and Letters. The instruments were formatted for Survey Monkey and the data collected electronically. The questionnaires were sent to the participants through the official channels mentioned above.

\section{RESULTS}

Internal consistency estimates of reliability were computed for each of the four factors. The alpha for the (23) items in Inhibition/Shyness was .90, the alpha for the (14) items in Lack of Time/Opportunity .88, the alpha for the (14) items in Social Repression .86, and the coefficient alpha for the (20) items in Lack of Motivation .89, all of which indicated satisfactory reliability.

The mean and standard deviations of each of the four factors were calculated to identify the most notable obstacles perceived by women in GCC. As Table (1) shows, the highest mean was found for the Lack of Time/Opportunities factor $(M=4.13, S D=.70)$, followed by the Lack of Motivation factor $(M=4.12, S D=.73)$, the Inhibition/Shyness factor $(M=3.76, S D=.69)$, and the Social Repression factor $(M=3.41, S D=.84)$. 


\section{Table 1}

Mean and standard deviation presented by GCC women in different factors of obstacles $(\mathrm{N}=297)$

\begin{tabular}{lcc}
\hline Obstacles & M & SD \\
\hline Inhibition/Shyness & 3.76 & .69 \\
Lack of Time/Opportunity & 4.13 & .70 \\
Social Repression & 3.41 & .84 \\
Lack of Motivation & 4.12 & .73 \\
\hline
\end{tabular}

A one-way Multivariate Analysis of Variance (MANOVA) was performed to determine the effect of age, education, country, income, marital status, field of study, and work sector on the four dependent variables (i.e., Lack of Time, Lack of Motivation, Inhibition/ Shyness, and Social Repression). Although the Wilks' Lambda showed no overall significant effect for age, Wilks' $\Lambda=.52, F(1,296)=1.12, p>.05$, partial $\eta^{2}=.15$, the follow up ANOVA showed that Inhibition/Shyness, $F(1,296)=1.49, p=.036$, partial $\eta^{2}=.19$, and Social Repression, $F(1,296)=1.45, p=.048$, partial $\eta^{2}=.185$ were significant.

Regarding education, although the Wilks' Lambda was not significant, Wilks' $\Lambda=.956, F(3,294)=1.643, p>.05$, partial $\eta^{2}=.022$, the ANOVA showed significant effects in Inhibition and Lack of Motivation respectively: $F(1,296)=3.857, p=.022$, partial $\eta^{2}=.026 ; F(1,296)=3.581, p=.029$, partial $\eta^{2}=.024$. Those studying/holding postgraduate degrees reported a higher mean with the Inhibition/Shyness factor $(M=3.90$, $S D=.63)$ compared with those studying/holding undergraduate degrees $(M=3.67$, $S D=.72)$. For the Lack of Motivation factor, those participants studying/holding a high school degree or below reported a higher mean $(M=4.33, S D=.52)$ compared with other groups.

As expected, the Wilks' Lambda was not significant when country was entered as an independent variable, Wilks' $\Lambda=.977, F(3,293)=.556, p>.05$, partial $\eta^{2}=.008$. None of the follow up ANOVAs was significant.

The fourth MANOVA was performed to examine the effects of field of study on the dependent variables. The results showed that participant obstacles to creativity differed by field of study. These differences were significant, Wilks' $\Lambda=.870, F(4,288)=2.05$, $p=.004$, partial $\eta^{2}=.034$. The follow up ANOVA showed that the only difference was found for the Social Repression factor: $F(5,291)=2.581, p=.026$, partial $\eta^{2}=.042$. Those in the arts reported having lower levels of obstacles related to social repression $(M=2.85, S D=.79)$ compared with those in engineering, who showed the highest mean $(M=3.62, S D=.69)$. 
Although income was expected to show a relationship with the dependent variables, especially Lack of Time/Opportunities, the MANOVA showed no significant differences in this regard, Wilks' $\Lambda=.967, F(3,291)=.820, p>.05$, partial $\eta^{2}=.011$. It was also hypothesized that marital status (married vs. single) might show some effects on some of the dependent variables; however, the results showed no significant differences due to social status, Wilks' $\Lambda=.979, F(3,295)=1.590, p>.05$, partial $\eta^{2}=.021$. In addition, no significant differences were found when sector (i.e., public, private, and business) was added as an independent variable, Wilks' $\Lambda=.974, F(3,293)=.947$, $p>.05$, partial $\eta^{2}=.013$.

All possible interactions between independent variables were examined to determine if two or more of the independent variables might show some effect on the four dependent variables. A significant interaction was found when education and sector were tested together as independent variables with the four dependent variables, Wilks' $\Lambda=.91, F(3,292)=1.77, p=.031$, partial $\eta^{2}=.024$; however, none of the follow up ANOVAs showed a significant effect.

Another significant interaction was found when country and sector were entered together as independent variables, Wilks' $\Lambda=.87, F(3,290)=1.62, p=.031$, partial $\eta^{2}=.033$. The follow up ANOVA showed that the only significant effect was found with the Lack of Motivation factor, $F(1,290)=1.23, p=.032$, partial $\eta^{2}=.047$. Participants from Bahrain reported the highest mean for the Lack of Motivation factor within the private sector $(M=4.58, S D=.34)$, and the lowest mean within the public sector $(M=3.99$, $S D=.89)$. Participants representing the United Arab Emirates reported the highest mean for the Lack of Motivation factor within business $(M=4.72, S D=.67)$, and the lowest mean within the private sector $(M=3.60, S D=.39)$. Participants from Saudi Arabia reported the highest mean for the Lack of Motivation factor within the public sector $(M=4.20, S D=.72)$, and the lowest mean within business $(M=3.82, S D=.55)$. The same trend was observed with participants representing Kuwait, with the highest mean within the public sector $(M=4.17, S D=.74)$, and lowest mean within business $(M=3.65, S D=.74)$.

A third significant interaction was found when income and field of study were added as independent variables, Wilks' $\Lambda=.77, F(3,281)=1.40, p=.035$, partial $\eta^{2}=.062$. The follow up ANOVA showed that the only significant effect was with the Inhibition/ Shyness factor, $F(1,281)=1.79, p=.044$, partial $\eta^{2}=.078$. Those who reported earning a salary of $600 \mathrm{BD}$ or below scored the highest mean for the Inhibition/Shyness factor within the business field $(M=4.02, S D=.29)$, and the lowest mean within the science 
field $(M=3.65, S D=.79)$. Those participants who reported earning a salary ranging between 601 to 1200 BD scored the highest mean for the Inhibition/Shyness factor within the business field as well $(M=4.13, S D=.46)$, but their lowest mean was within the maths field $(M=3.10, S D=.87)$. Those who reported earning a salary ranging between 1201 to 1800 BD scored the highest mean for the Inhibition/Shyness factor within the Engineering field $(M=4.29, S D=.27)$, and the lowest mean within the arts field $(M=3.04, S D=.18)$. Finally, those participants who reported earning a salary above 1800 BD scored the highest mean for the Inhibition/Shyness factor within the maths field $(M=4.02, S D=.32)$, whereas the lowest mean was observed in the science field $(M=3.57, S D=.59)$.

A fourth interaction was observed when marital status and sector were added as independent variables, Wilks' $\Lambda=.94, F(4,291)=2.29, p=.02$, partial $\eta^{2}=.031$. None of the follow up ANOVAs were significant.

Finally, a fifth significant interaction was observed when field of study and sector were added as independent variables, Wilks' $\Lambda=.83, F(3,294)=1.60, p=.019$, partial $\eta^{2}=.044$. None of the follow up ANOVAs were significant. All other interactions between independent variables and the four Obstacle factors were not significant.

When comparing the results of this study with two other studies conducted with the same instrument, utilizing Brazilian and Mexican samples, women in GCC reported significantly more obstacles, across all four factors (see Table 2). Table (2) presents the means of each sample, as well as the $t$ test values for each comparison (i.e., the current study vs other the two studies in each factor).

Table 2

Comparison of mean differences in the current study with Brazilian and Mexican women samples

\begin{tabular}{|c|c|c|c|c|c|c|c|c|}
\hline \multirow[t]{2}{*}{ Obstacles } & \multicolumn{2}{|c|}{$\begin{array}{l}\text { Current Study } \\
\qquad(\mathrm{n}=297)\end{array}$} & \multicolumn{2}{|c|}{$\begin{array}{c}\text { Alencar et al. (2003) } \\
\qquad(n=269)\end{array}$} & \multicolumn{2}{|c|}{$\begin{array}{c}\text { Alencar }(2001) \\
(n=135)\end{array}$} & \multirow{2}{*}{$\begin{array}{c}\begin{array}{c}\text { Current vs } \\
\text { Alencar et al. } \\
(2003)\end{array} \\
t(564)\end{array}$} & \multirow{2}{*}{$\begin{array}{c}\begin{array}{c}\text { Current vs } \\
\text { Alencar } \\
(2001)\end{array} \\
t(430)\end{array}$} \\
\hline & $M$ & $\mathrm{SD}$ & M & SD & M & SD & & \\
\hline Inhibition/Shyness & 3.76 & .69 & 3.64 & .73 & 3.61 & .76 & $2.00^{*}$ & 1.96 \\
\hline $\begin{array}{l}\text { Lack of Time/ } \\
\text { Opportunity }\end{array}$ & 4.13 & .70 & 3.64 & .74 & 3.71 & .77 & $8.07^{* *}$ & $5.40^{\star \star}$ \\
\hline Social Repression & 3.41 & .84 & 3.08 & .70 & 3.10 & .77 & $5.09^{* *}$ & $3.77^{* *}$ \\
\hline Lack of Motivation & 4.12 & .73 & 3.44 & .81 & 3.32 & .85 & $10.45^{\star \star}$ & $9.46^{\star \star}$ \\
\hline
\end{tabular}




\section{DISCUSSION}

The main objective of this study was to identify the most notable blocks to personal creativity among GCC women. As noted earlier, a search of the main databases in Arabic, including Dar Al-Mandumahand E-Marifah, showed an absence of relevant studies related to personal creativity among women. Addressing the conditions that prevent women from the full expression of their life is important.

It was not surprising that lack of time and opportunities were the most significant obstacles for GCC women. In their discussion of gender and creativity, Runco et al. (2010) described how time was an issue for most women. This is especially true in Arab culture, where women are expected to get married at an early age, have five to six children, and take the responsibility for an extended family. Runco et al. (2010) also pointed out that opportunities are related to expectations. As they put it, "If a woman is not expected to do creative things, will anyone notice and support her if she is in fact creative?" (p. 344).

What was not expected was the high mean found within the Lack of Motivation factor. This was a disappointing finding about women in the GCC that needs to be further studied. Why would GCC women report low levels of motivation to be creative? There are different explanations for this result, but first it is important to specify which kind of motivation is assessed by the Obstacles to Personal Creativity Inventory. An examination of the 20 items in this factor shows that the focus is on intrinsic motivation. Some examples are "I will be more creative if...1) I were more enthusiastic, 2) I were more curious, 3) I were more persistent, 4) I were not so laid back, and 5) I had more motivation to create" (Alencar, 2014, p. 31). Social expectations might again explain the present results, especially if we consider the participants' age (i.e., adults). It may be that the motivation to be creative is inhibited, especially in this part of the world where a woman recognizes that her role in society is to get married and raise children. A second explanation is related to the educational system and how it reinforces/inhibits creativity and creative thinking. Consider in this regard the 2016 annual report of Bahrain Education and Training Quality Authority. The term "creativity" was mentioned only twice throughout the 142 page report, and these two hits were titles for conference papers. However, this explanation would probably apply to both male and female students. A third explanation reflects the possibility that creativity is not well understood. Some countries view ability as innate rather than a matter of effort, and it could be that the women in this sample responded from the perspective that creativity is an ability and is not influenced by motivation. As suggested above, this issue needs to be further investigated to locate the sources or factors that contribute to having low motivation toward creativity. One suggestion to better understand 
this issue might be through conducting case studies and interviews with a random sample of women in GCC, and with examples of some creative women as well.

Although the mean scores for the Inhibition/Shyness and Social Repression factors were lower than the mean scores for the Lack of Time/Opportunities and Lack of Motivation factors in the current study, these means were higher than the mean scores reported in the comparative studies: Alencar et al. (2003) and Alencar (2001) (see Table 2).

It was hypothesized that there would be no significant differences among the nationalities sampled here in the four obstacles assessed by the Obstacles to Personal Creativity Inventory. The results supported this hypothesis. In some ways, the GCC is a homogenous group. Families and clans are distributed throughout the six countries. Language, traditions and costumes, religious, and socioeconomic status are some examples of commonalities among these countries.

Regarding education, the findings suggest that having higher educational degrees might be beneficial for the motivation toward creativity. Those who reported having lower degrees (i.e., high school) scored higher on the Lack of Motivation factor.

It was expected that participants from different fields of study might differ in the levels of each factor or blocks to personal creativity. This was only true for the Social Repression factor. Those in artistic fields reported having lower levels for social repression compared with those in engineering. It makes sense that those studying or working in artistic fields might show lower levels of social repression since art is about self-expression, and this (i.e., self-expression), might help to reduce the levels of social repression. It also makes sense that females working in engineering might show higher levels of social repression since this field of study (i.e., engineering) is known to be male-dominated.

\section{Limitations and Future Directions}

As with many studies in social sciences, sampling is always an issue. Although we greatly appreciate the clearance from the official institutions in the GCC region, such as the Supreme Council for Women in Bahrain, the sample was only moderate, with 300 participants. Moreover, we could not reach any participants in Oman and Qatar because of some logistic and communication issues.

It is important to note that the current study relied on one format of instrument (a self -report) and focused only on one kind of obstacle related to creativity (i.e., personal). Future studies might consider looking at other obstacles such as those related to the work place and environmental blocks to creativity and creative thinking. However, the main recommendation related to the current study's findings is to investigate the reasons behind the lack of motivation reported by the study subjects and to create an encouraging environment for females to show their creative strengths, which in turn will benefit the whole of society. 


\section{REFERENCES}

Al-Ahmadi, H. (2011). Challenges facing women leaders in Saudi Arabia. Human Resources Development International, 14(2), 149-166.

Alencar, E. M. L. S. (2001). Obstacles to personal creativity among university students. Gifted Education International, 15(2), 133-140.

Alencar, E. M. L. S., De Souza Fleith, D., \& Martinez, A. M. (2003). Obstacles to personal creativity between Brazilian and Mexican university students: A comparative study. Journal of Creative Behavior, 37(3), 179-192. doi:10.1002/j.2162-6057.2003.tb00832.x

Alencar, E. M. L. S. (2014). Obstacles to Personal Creativity Inventory. In E. M. L. S. Alencar, M. D. F. Bruno-Faria, \& D. S. Fleith (Eds.), Theory and practice of creativity measurement (pp.21-35). Waco, TX: Prufrock.

Baer, J., \& Kaufman, J. C. (2008). Gender differences in creativity. Journal of Creative Behavior, 42(2), 75-105.

Bahrain Education and Training Quality Authority. (2016). Annual report. Retrieved from http://www.bqa.gov.bh/En/Mediacenter/Documents/BQA\%20Annual\%20Report\% 202016\%20(English).PDF

Cattell, J. M. (1903). A statistical study of eminent men. Popular Science Monthly, 62, 359-377.

Coffman, J. (2003). The Gulf: Privatization and Americanization. International Higher Education, (33), 17-19.

Cramond, B. (2011). Women and creativity. In M. A. Runco \& S. R. Pritzker (Eds.), Encyclopedia of creativity, (pp. 521-524). San Diego, CA: Academic Press.

Diamond, A. M. (1986). The life-cycle research productivity of mathematicians and scientists. Journal of Gerontology, 41(4), 520-525.

He, W., \& Wong, W. (2011). Gender differences in creative thinking revisited: Findings from analysis of variability. Personality and Individual Differences, 51, 807-811. doi:10.1016/j.paid.2011.06.027

Isaksen, S. G., Lauer, K. J., Ekvall, G. and Britz, A. (2000-2001). Perceptions of the best and worst climates for creativity: Preliminary validation evidence for the Situational Outlook Questionnaire. Creativity Research Journal, 13, 171-184.

Karwowski, M., Jankowska, D. M., Gajda, A., Marczak, M., Groyecka, A., \& Sorokowski, P. (2016). Greater male variability in creativity outside the WEIRD world. Creativity Research Journal, 28(4), 467-470. 
Mostafa, M. M., \& El-Masry, A. (2008). Perceived barriers to organizational creativity: A cross-cultural study of British and Egyptian future marketing managers. Cross Cultural Management, 15(1), 81-93. doi:10.1108/13527600810848845

Pagnani, A. (2011). Gender differences. In M. A. Runco \& S. R. Pritzker (Eds.), Encyclopedia of creativity (pp. 551-557). San Diego, CA: Academic Press.

Reis, S. M. (2002). Toward a theory of creativity in diverse creative women. Creativity Research Journal, 14, 305-316.

Rickards, T., \& Jones, L. J. (1991). Toward the identification of situational barriers to creative behaviors: The development of a self report inventory. Creativity Research Journal, 4, 303-316.

Rosenberry, K. (2005). Organizational barriers to creativity in law schools and the legal profession. California Western Law Review, 41(2), 423-457.

Runco, M.A., Cramond, B., \& Pagnani, A. R. (2010). Sex differences in creative potential and creative performance. In J. C. Chrisler\& D. McCreary (Eds.), Handbook of gender research in psychology (vol. 2, pp. 343-357). New York: Springer.

Sadi, M. A., \& Al-Dubaisi, A. H. (2008). Barriers to organizational creativity: The marketing executives' perspective in Saudi Arabia. Journal of Management Development, 27(6), 574-599.

Simonton, D. K. (1984). Artistic creativity and interpersonal relationships across and within generations. Journal of Personality \& Social Psychology, 46(6), 1273-1286.

Simonton, D. K. (2000). Creativity: Cognitive, personal, developmental, and social aspects. The American Psychologist, 55(1), 151-158.

Simonton, D. K. (2016). Scientific genius in Islamic civilization: Quantified time series from qualitative historical narratives. Journal of Genius and Eminence, 1(1), 4-13.

Simonton, D. K. (2018). Intellectual genius in the Islamic golden age: Cross-civilization replications, extensions, and modifications. Psychology of Aesthetics Creativity and the Arts, 12(2), 125-135.

Taqi, N. (2016). The challenges facing women entrepreneurs: A study on Gulf Cooperation Council (GCC) countries (Unpublished doctoral dissertation). Brunel University, London.

United Nations. (2017). Household size and composition around the world 2017. Retrieved from http://www.un.org/en/development/desa/population/publications/pdf/ ageing/household_size_and_composition_around_the_world_2017_data _booklet.pdf 
Walter, C. (2012). Work environment barriers prohibiting creativity. Procedia - Social and Behavioral Sciences, 40 (Asia Pacific Business Innovation and Technology Management Society), 642-648. doi:10.1016/j.sbspro.2012.03.243

Witt, L. A., \& Beorkrem, M. N. (1989). Climate for creative productivity as a predictor of research usefulness and organizational effectiveness in an $R \& D$ organization. Creativity Research Journal, 2, 30-40.

Corresponding author at: Ahmed M. Abdulla, PhD., Arabian Gulf University, Road 2904, Building 293, Manama 329, Bahrai.

E-mail: ahmedmda@agu.edu.bh 